\title{
BOUNDS FOR DETERMINANTS WITH DOMINANT PRINCIPAL DIAGONAL ${ }^{1}$
}

\section{G. BALEY PRICE}

1. Introduction. This note establishes upper and lower bounds for determinants with dominant principal diagonal (see a recent paper on these determinants by Olga Taussky [9] $\left.{ }^{2}\right)$. The lower bound is an improvement of a similar lower bound given by Ostrowski [6]. The best known theorems concerning upper bounds of determinants are Nanson's theorem [5] and the generalizations of it proved by Szász [8], Fischer [1], and others (see [2, pp. 34-36]), and Hadamard's Determinant Theorem (see [4, pp. 90-101]). H. von Koch [3] gave a lower bound for a determinant, and Ostrowski $[6 ; 7]$ has given both upper and lower bounds. Previous results are better in some cases, but the bounds in this note are closer in many others. The bounds given here are recommended also by their simplicity.

2. The theorems. Let $\left(a_{i j}\right)$ denote an $n \times n$ matrix and $D$ its determinant $\left|a_{i j}\right|$. Set

$$
\begin{gathered}
A_{i}=\sum_{j=1, j \neq i}^{n}\left|a_{i j}\right|, \quad m_{i}=\left|a_{i i}\right|-\sum_{j=i+1}^{n}\left|a_{i j}\right| \\
M_{i}=\left|a_{i i}\right|+\sum_{j=i+1}^{n}\left|a_{i j}\right|
\end{gathered}
$$

for $i=1,2, \cdots, n$.

TheOREM 1. Let $\left(a_{i j}\right)$ be an $n \times n$ matrix with complex elements such that.

$$
\left|a_{i i}\right|>A_{i}, \quad i=1,2, \cdots, n .
$$

Then

$$
0<m_{1} m_{2} \cdots m_{n} \leqq|D| \leqq M_{1} M_{2} \cdots M_{n} .
$$

An equality holds in (2) if and only if

$$
a_{i j}=0, \quad j>i .
$$

If (3) holds, then

Presented to the Society, November 24, 1950; received by the editors June 26, 1950.

${ }^{1}$ The results in this. note were obtained in connection with work on Project No. NR 043093 of the Office of Naval Research.

. 2 Numbers in brackets refer to the bibliography at the end of the paper. 


$$
0<\left|a_{11} a_{22} \cdots a_{n n}\right|=m_{1} m_{2} \cdots m_{n}=|D|=M_{1} M_{2} \cdots M_{n} .
$$

ThEOREM 2. Let $\left(a_{i j}\right)$ be an $n \times n$ matrix with real elements such that

$$
a_{i i}>A_{i}, \quad i=1,2, \cdots, n .
$$

Then

$$
0<m_{1} m_{2} \cdots m_{n} \leqq D \leqq M_{1} M_{2} \cdots M_{n} .
$$

An equality holds in (5) if and only if (3) holds. If (3) holds, then

$$
0<a_{11} a_{22} \cdots a_{n n}=m_{1} m_{2} \cdots m_{n}=D=M_{1} M_{2} \cdots M_{n} \text {. }
$$

The theorems of Nanson [5] and Ssász [8] yield better upper bounds in certain cases as stated in the following theorem.

TheOREM 3. Let $\left(a_{i j}\right)$ be an $n \times n$ Hermitian matrix such that $a_{i i}>A_{i}$ for $i=1,2, \cdots, n$. Then

$$
\begin{gathered}
0<m_{1} m_{2} \cdots m_{n} \leqq D, \\
D=P_{n}^{p_{n}} \leqq P_{n-1}^{p_{n}-1} \leqq \cdots \leqq P_{r}^{p_{r}} \leqq \cdots \leqq P_{2}^{p_{2}} \leqq P_{1}^{p_{1}} \\
=a_{11} a_{22} \cdots a_{n n},
\end{gathered}
$$

where $P_{r}$ denotes the product of all the rth order principal minors of $D$ and $p_{r}=1 / C(n-1, r-1)$ for $r=1,2, \cdots, n$.

Szász has shown that this theorem can be extended as follows. It may be that $\left(a_{i j}\right)$ does not satisfy the hypotheses of Theorem 3 , but that nonzero constants $c_{1}, c_{2}, \cdots, c_{n}$ can be found such that the new matrix $\left(a_{i j} c_{i} / c_{j}\right)$ does. Then $D=\left|a_{i j}\right|$ has all of its principal minors positive and satisfies (7). An extension of (6) will be given below.

Let $\left(a_{i j}\right)$ be a matrix which satisfies the hypotheses of one of the three theorems. If the same permutation is applied to the rows and to the columns of $\left(a_{i j}\right)$, the new matrix satisfies the hypotheses of the same theorem. In this way new upper and lower bounds $M_{1}^{\prime} M_{2}^{\prime} \cdots M_{n}^{\prime}$ and $m_{1}^{\prime} m_{2}^{\prime} \cdots m_{n}^{\prime}$ are obtained for $D$ or $|D|$, and in general these are different from the original bounds. Since there are $n$ ! permutations of the rows and columns, the theorems yield $n$ ! upper and lower bounds. The best upper bound (lower bound) that can be obtained from the theorems is thus the minimum (maximum) of the $n$ ! upper bounds (lower bounds).

It is possible to extend Theorems 1,2 , and 3 to those cases in which $\left|a_{i i}\right|>A_{i}$ and $a_{i i}>A_{i}$ are replaced by $\left|a_{i i}\right| \geqq A_{i}$ and $a_{i i} \geqq A_{i}$. Form new determinants by changing the elements on the principal 
diagonal so that the original inequalities hold. Then the usual continuity arguments show that (2) becomes

$$
0 \leqq m_{1} m_{2} \cdots m_{n} \leqq|D| \leqq M_{1} M_{2} \cdots M_{n},
$$

that (5) becomes

$$
0 \leqq m_{1} m_{2} \cdots m_{n} \leqq D \leqq M_{1} M_{2} \cdots M_{n}
$$

and that (6) becomes

$$
0 \leqq m_{1} m_{2} \cdots m_{n} \leqq D .
$$

It is not difficult to determine the conditions under which the equality holds at the extreme left in these three inequalities, but the results will not be stated here (in this connection see Olga Taussky $[9$, p. 673]).

A significant extension of the above results will now be illustrated in the case of Theorem 1. Consider a set of determinants $D_{1}, D_{2}, \cdots$, $D_{n-1}$, where $D_{k}$ is formed from the determinant $\left|a_{i j}\right|(i, j=k, \cdots, n)$ by dividing the first column by the positive constant $r_{k}$. Set

$$
m_{k}^{*}=\left|a_{k b}\right|-r_{k} \sum_{j=k+1}^{n}\left|a_{k j}\right|, \quad M_{k}^{*}=\left|a_{k k}\right|+r_{k} \sum_{j=k+1}^{n}\left|a_{k j}\right|,
$$

for $k=1,2, \cdots, n-1$, and set $m_{n}^{*}=M_{n}^{*}=\left|a_{n n}\right|$.

TheOREM 4. Let $\left(a_{i j}\right)$ be an $n \times n$ matrix with complex elements. If there exists a set of positive constants $r_{1}, r_{2}, \cdots, r_{n-1}$ such that each of the determinants $D_{1}, D_{2}, \cdots, D_{n-1}$ is a determinant with dominant principal diagonal, that is, such that the absolute value of each term on the principal diagonal of $D_{k}$ is greater than the sum of the absolute values of all other terms in the same row, then

$$
0<m_{1}^{*} m_{2}^{*} \cdots m_{n}^{*} \leqq|D| \leqq M_{1}^{*} M_{2}^{*} \cdots M_{n}^{*}
$$

An equality holds if and only if (3) holds; and if (3) holds, (2') holds.

Theorem 4 can be employed in the following manner. It may be that $D$ does not satisfy the hypotheses of Theorem 1, but that $r_{1}$ can be chosen so that $D_{1}$ does. The best bounds will be obtained by choosing $r_{1}$ as small as possible. If $D_{1}$ is a determinant with dominant principal diagonal, then $D_{2}, \cdots, D_{n-1}$ are certainly determinants of the same type for $r_{2}=\cdots=r_{n-1}=1$. Once again, however, the bounds will be improved if smaller values can be chosen for these constants.

3. Lemmas. The theorems of the last section can be proved most easily with the aid of two lemmas which will be given in this section. 
LEMMA 1. If $\left(a_{i j}\right)$ is any matrix with complex elements such that $\left|a_{i i}\right|$ $>A_{i}$ for $i=1,2, \cdots, n$, then $D=\left|a_{i j}\right| \neq 0$.

The result stated in this lemma has been discovered and published many times. A simple proof and an extensive bibliography will be found in [9].

LEMMA 2. Let a system of equations

$$
a_{i 1}+\sum_{j=2}^{n} a_{i j} x_{j}=0, \quad i=2,3, \cdots, n,
$$

be given in which

$$
\left|a_{i i}\right|>(1 / r)\left|a_{i 1}\right|+\sum_{j=2, j \neq i}^{n}\left|a_{i j}\right|, \quad i=2,3, \cdots, n,
$$

where $r$ is some number greater than zero. Then the system (9) has a unique solution $x_{2}, \cdots, x_{n}$, and

$$
\max \left(\left|x_{2}\right|,\left|x_{3}\right|, \cdots,\left|x_{n}\right|\right)<r \text {. }
$$

To prove the lemma, observe that

$$
\left|a_{i i}\right|>\sum_{j=2, j \neq i}^{n}\left|a_{i j}\right|, \quad i=2,3, \cdots, n,
$$

by (10). Then the determinant of the system of equations (9) is a determinant with dominant principal diagonal, and its value is not zero by Lemma 1. Thus (9) has a unique solution. Assume now that (11) is false. Let $\left|x_{i}\right|=\max \left(\left|x_{2}\right|,\left|x_{3}\right|, \cdots,\left|x_{n}\right|\right) \geqq r$. Then from (9) it follows that

$$
\begin{aligned}
-a_{i i} x_{i} & =a_{i 1}+\sum_{j=2, j \neq i}^{n} a_{i j} x_{j}, \\
\left|a_{i i}\right|\left|x_{i}\right| & \leqq\left|a_{i 1}\right|+\sum_{j=2, j \neq i}^{n}\left|a_{i j}\right|\left|x_{j}\right|, \\
\left|a_{i i}\right| & \leqq \frac{\left|a_{i 1}\right|}{\left|x_{i}\right|}+\sum_{j=2, j \neq i}^{n}\left|a_{i j}\right| \frac{\left|x_{j}\right|}{\left|x_{i}\right|}, \\
\left|a_{i i}\right| & \leqq(1 / r)\left|a_{i 1}\right|+\sum_{j=2, j \neq i}^{n}\left|a_{i j}\right|,
\end{aligned}
$$

since $\left|x_{i}\right| \geqq r$ and $\left|x_{j}\right| /\left|x_{i}\right| \leqq 1$. But (12) contradicts (10), and this contradiction establishes (11). The proof of Lemma 2 is complete.

4. Proofs of the theorems. Let $\left(a_{i j}\right)$ be a matrix which satisfies the 
hypotheses of Theorem 1. Consider the system of equations (9) with the coefficients taken from $\left(a_{i j}\right)$. Then from (1) it follows that (10) is satisfied with $r=1$. Let the solution of (9) in this case be $x_{2}^{(1)}, x_{3}^{(1)}, \cdots, x_{n}^{(1)}$. Multiply the $j$ th column of $D$ by $x_{j}^{(1)}, j=2,3, \cdots$, $n$, and add to the first column. As a result of (9) it follows that

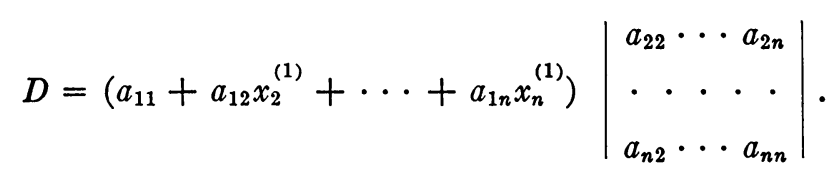

The determinant which appears on the right here satisfies the same hypotheses as $D$, and the reduction can be repeated until the following evaluation is obtained:

$$
\begin{aligned}
& D=\left(a_{11}+a_{12} x_{2}^{(1)}+\cdots+a_{1 n} x_{n}^{(1)}\right)\left(a_{22}+a_{23} x_{3}^{(2)}+\cdots\right. \\
& \left.+a_{2 n} x_{n}^{(2)}\right) \cdots a_{n n} \text {. }
\end{aligned}
$$

Since $\left|x_{j}^{(k)}\right|<1$ for all $k$ and $j$, and since $m_{j}>0$ for all $j$ by (1), the conclusions in (2) and $\left(2^{\prime}\right)$ follow by the use of standard inequalities for complex numbers. The proof of Theorem 1 is complete, and the proof of Theorem 2 is entirely similar.

To prove Theorem 3, observe first that the value of a Hermitian matrix is real. Since both determinants in (13) are Hermitian and hence real, it follows that the parenthesis on the right is real. Since $a_{11}$ is real, it follows that $a_{12} x_{2}^{(1)}+\cdots+a_{1 n} x_{n}^{(1)}$ is real. Since $a_{i i}>A_{i}$ and $\left|x_{j}^{(1)}\right|<1$ for $j=2, \cdots, n$, the first factor on the right in (14) is positive and not less than $m_{1}$. Similar considerations applied to the other factors in (14) complete the proof of (6).

To prove (7) observe, first, that every principal minor of $D$ is itself Hermitian; and, second, that every principal minor has a dominant principal diagonal as a result of the hypothesis $a_{i i}>A_{i}$ for $D$. Then all principal minors of $D$ are positive by (6). Finally, Szász [8] has established the inequality (7) for any Hermitian matrix all of whose principal minors are positive. The proof of Theorem 3 is complete.

To prove Theorem 4, observe that $D=r_{1} D_{1}$. Since $D_{1}$ satisfies the hypotheses of Theorem 1, it is possible to apply the reduction indicated in (13) to obtain

$$
D=r_{1}\left(\frac{a_{11}}{r_{1}}+a_{12} x_{2}^{(1)}+\cdots+a_{1 n} x_{n}^{(1)}\right) r_{2} D_{2} .
$$

Since $D_{2}, D_{3}, \cdots, D_{n-1}$ satisfy the same hypotheses as $D_{1}$, the re- 
duction can be continued to obtain

$$
\begin{aligned}
D= & {\left[a_{11}+r_{1}\left(a_{12} x_{2}^{(1)}+\cdots+a_{1 n} x_{n}^{(1)}\right)\right] } \\
\cdot & {\left[a_{22}+r_{2}\left(a_{23} x_{3}^{(2)}+\cdots+a_{2 n} x_{n}^{(2)}\right)\right] } \\
& \cdots\left[a_{n-1} n+1+r_{n-1} a_{n-1} x_{n}^{(n-1)}\right] a_{n n} .
\end{aligned}
$$

The inequality in (8) now follows from this equation in the usual way. The proof of Theorem 4 is complete.

The same considerations can be used to prove an extension of Theorem 2 and of equation (6) in Theorem 3 similar to the extension of Theorem 1 given by Theorem 4 .

Finally, it may be remarked that the results of this section suggest a simple and independent proof of Lemma 1 by the method of mathematical induction.

\section{BIBLIOGRAPHY}

1. E. Fischer, Ueber den Hadamardschen Determinantensatz, Archiv der Mathematik und Physik (3) vol. 13 (1908) pp. 32-40.

2. G. H. Hardy, J. E. Littlewood, and G. Polya, Inequalities, Cambridge University Press, 1934.

3. Helge von Koch, Über das Nichtverschwinden einer Determinante nebst Bemerkungen ïber Systeme unendlich vieler linearer Gleichungen, Jber. Deutschen Math. Verein. vol. 22 (1913) pp. 285-291.

4. Thomas Muir, Contributions to the history of determinants 1900-1920, London, Blackie, 1930.

5. E. J. Nanson, $A$ determinant inequality, Messenger of Mathematics vol. 31 (1901-1902) pp. 48-50.

6. A. Ostrowski, Sur la détermination des bornes inférieures pour une classe des dêterminants, Bull. Sci. Math. (2) vol. 61 (1937) pp. 19-32.

7. - Über die Determinanten mit überwiegender Hauptdiagonale, Comment. Math. Helv. vol. 10 (1937) pp. 69-96.

8. Otto Szász, Über eine Verallgemeinerung des Hadamardschen Determinantensatzes, Monatshefte für Mathematik und Physik vol. 28 (1917) pp. 253-257.

9. Olga Taussky, A recurring theorem on determinants, Amer. Math. Monthly vol. 56 (1949) pp. 672-676.

UNIVERSITY OF KANSAS 